\title{
Endoscopic Ultrasound-Guided Fine Needle Aspiration Accurately Diagnoses Smaller Pancreatic Neuroendocrine Tumors Compared To Computer Tomography-Guided Fine Needle Aspiration
}

\author{
Jeremy Wang ${ }^{1,4}$, Jihane N Benhammou ${ }^{1,2,4}$, Kevin Ghassemi ${ }^{2}$, Stephen Kim ${ }^{2,4}$, Alireza Sedarat ${ }^{2,4}$, \\ James Farrell $^{3}$ and Joseph R Pisegna ${ }^{4,5 *}$ \\ ${ }^{1}$ Department of Medicine, David Geffen School of Medicine, Los Angeles, California, USA \\ ${ }^{2}$ Division of Digestive Diseases, David Geffen School of Medicine, Los Angeles, California, USA \\ ${ }^{3}$ Division of Digestive Diseases, Yale University School of Medicine New Haven, Connecticut, USA \\ ${ }^{4}$ Division of Gastroenterology, Hepatology and Parenteral Nutrition, VA Greater Los Angeles Healthcare System, Los Angeles, California, USA \\ ${ }^{5}$ Department of Human Genetics, David Geffen School of Medicine, Los Angeles, California, USA
}

Received: January 20, 2017; Accepted: March 7, 2017; Published: March 27, 2017

*Corresponding author: Joseph R. Pisegna, Chief, Division of Gastroenterology, Hepatology and Parenteral Nutrition, VA Greater Los Angeles Healthcare System (691/111C), 11301 Wilshire Blvd, Los Angeles, CA 90073, USA, Tel: 310-268-3578; Fax: 310-268-4096; E-mail: joseph.pisegna@va.gov

\begin{abstract}
Introduction: The role of EUS-guided FNA as a highly sensitive modality in the diagnosis of pancreatic adenocarcinoma is well documented. However, there is little published data on the role of EUS-FNA in diagnosing pancreatic neuroendocrine tumors (NETs).
\end{abstract}

Objective: The aim of this study is to compare the sensitivity of EUS-FNA to that of CT-FNA for diagnosing pancreatic NETs.

Methods: This is a single institution retrospective analysis of the operating characteristics of EUS-FNA and CT-FNA in detecting pancreatic NETs. Only patients with a final diagnosis of pancreatic NET were selected for this study. Procedure related data, including tumor size and location, and presence of a cytotechnologist were recorded. The results of each FNA were compared to the final clinicopathological diagnosis to calculate sensitivity.

Results: Twenty-eight patients undergoing FNA (19 by EUS, 9 by CT) were analyzed. NETs diagnosed by EUS-FNA were smaller compared with CT-FNA $(2.7 \pm 0.9 \mathrm{~cm}$ vs. $6.5 \pm 2.1 \mathrm{~cm}, \mathrm{p}=0.009)$ and were more often found in the pancreatic head $(47.4 \%$ vs. $11.1 \%, p=$ $0.035)$. There were no significant differences in sensitivity between EUS-FNA and CT-FNA specimens (73.7\% vs. $88.9 \%, \mathrm{p}=0.33$ ).

Conclusion: EUS-guided FNA is as sensitive as CT-guided FNA in diagnosing pancreatic NETs, but its main advantage is in the diagnosis of smaller pancreatic NETs in the head of the pancreas. It may also be the preferred approach in the diagnosis of multifocal pancreatic NETs in the setting of MEN I Syndrome.

Key Words: Endoscopic Ultrasound; Computed Tomography; Fine Needle Aspiration; Pancreatic Neuroendocrine Tumor

\section{Introduction}

Neuroendocrine tumors (NETs) include a broad group of pancreatic neoplasms, including insulinoma, gastrinoma, glucagonoma, somatostatinoma, and carcinoid tumors. Patients with NETs usually present with symptoms related to the hypersecretion of peptides or biogenic amines which also help to characterize the particular pancreatic NET. However, 15$30 \%$ of NETs are nonfunctioning, and these may be discovered incidentally in asymptomatic patients, or they may present as a symptomatic mass [1]. Pancreatic NETs, in addition to parathyroid adenomas and pituitary tumors, can be found in the setting of multiple endocrine neoplasia type 1 (MEN-1) disease, occurring in $30-75 \%$ of MEN-1 patients when assessed by clinical screening methods, and approaching $100 \%$ in autopsy series [2].

Compared to the poor long-term survival seen with adenocarcinoma of the pancreas, the overall five-year survival for patients with pancreatic NETs is $\sim 60-97 \%$, depending on the type of NET and whether there is an association with MEN-1 [3]. For patients with MEN-1, the overall ten-year survival is $94 \%$ [2]. Therefore, making a tissue diagnosis on a pancreatic lesion can convey valuable prognostic information and help determine when surgical intervention might be curative.

Endoscopic ultrasound (EUS) has been shown to be a highly accurate technique for the visualization of small functioning neuroendocrine tumors not evident on computed tomography (CT) and for detecting the presence of multiple lesions in patients with MEN-1 syndrome [4]. Furthermore, endoscopic ultrasoundguided fine needle aspiration (EUS-FNA) has shown high safety, sensitivity, specificity, and accuracy in the diagnosis of general pancreatic lesions and pancreatic NETs [5-26]. Previous studies have shown that EUS-FNA demonstrates comparable accuracy relative to ultrasound-guided fine needle aspiration (US-FNA) and computed tomography-guided fine needle aspiration(CTFNA) in the diagnosis of general pancreatic lesions, and resulted in greater accuracy than both in evaluating lesions less than $3 \mathrm{~cm}$ 
after controlling for location $[5,6]$. However, few studies have evaluated the accuracy of EUS-FNA compared to US-FNA or CTFNA in the diagnosis of PNETs specifically. Only one prior, smaller study evaluated EUS-FNA and CT-FNA of endocrine tumors of the pancreas, but its objective was to compare the cytologic features of islet cell tumors obtained by these two modalities, and no statistical comparison was made [27]. The present study aims to compare the operating characteristics of EUS-FNA and CT-FNA in the diagnosis of pancreatic NETs.

\section{Methods}

\section{Patient Identification}

This is a single institution, retrospective study of the operating characteristics of EUS-FNA and CT-FNA in diagnosing pancreatic NETs. We used a computer-generated cytology database to identify patients who had undergone FNA of the pancreas at the UCLA Medical Center from January 1992 to April 2006. A large date range was used in order to maximize the number of cases that could be included in this study. While fine needle aspiration is not an uncommon procedure, our inclusion criteria required a final diagnosis of neuroendocrine tumor, a much rarer diagnosis. These patients' records were reviewed to determine: (1) The modality used to perform the FNA, (2) A cytologic diagnosis, if one was made, and (3) A final diagnosis of the pancreatic lesion, if available. All patients with a final diagnosis of a pancreatic NET, whether or not cytologic diagnosis was made, were included in the study. A final diagnosis of a pancreatic NET was made based on at least one of the following criteria: (1) Histopathology of a surgical resection specimen or CT-guided core biopsy, (2) A known history of MEN-1, (3) Follow-up of at least 12 months to assess for new manifestations or progression of disease, or (4) Cytological diagnosis of NET from the FNA itself. This last criterion was used only if none of the first three criteria could be met. We assumed $100 \%$ specificity for the FNA diagnosis of pancreatic NET based on prior use of this methodology [28].

For each case, the medical record was reviewed and cytopathologic, imaging, and procedural data were collected. The procedure report was reviewed to record the characteristics of the lesion, number of FNA passes, evidence of local mass invasion and, in the case of EUS, whether a cytotechnologist was present during the procedure to determine specimen adequacy. Size was recorded based on the longest dimension of the mass. Location was categorized as head/uncinate, body, or tail. Radiologic reports were used to assess location and size of the pancreatic lesions, as well as for evidence of local and/or distant metastases.

\section{EUS-guided and CT-guided FNA techniques}

EUS was performed using a Pentax (Pentax Precision Instruments Corporation, Orangeburg, New York) or Olympus (Olympus America, Melville, New York) linear array echoendoscope (5.0-7.5 MHz). After EUS localization, fine needle aspiration was performed using a 22-gauge needle (Wilson-Cook Medical Incorporated, Winston-Salem, North Carolina) either transgastrically or transduodenally (Figure
1). CT-guided FNA was performed by either direct coaxial or tandem needle technique ${ }^{28}$ using a 22 -gauge needle (Somatex Medical Technologies GmbH, Teltow, Germany) (Figure 2). A cytotechnologist was present during all CT-FNA procedures to assure specimen adequacy.

\section{Cytologic Material Preparation and Examination}

Cytologic material was placed onto a glass slide and thin smears were prepared. If a cytotechnologist was present during the procedure, the sample was stained immediately using the rapid Papanicolau technique. The presence or absence of a cytotechnologist during EUS was determined by whether the endosonographer felt beforehand that a particular case would benefit from their presence. All cytologic specimens were interpreted by a staff cytopathologist, and these reports were used in this study (Figures 3 and 4). In this study, only specimens specifically called NET on cytology were deemed positive. All other non-benign readings (atypia, suspicious, non-diagnostic) were considered negative cytologic results.

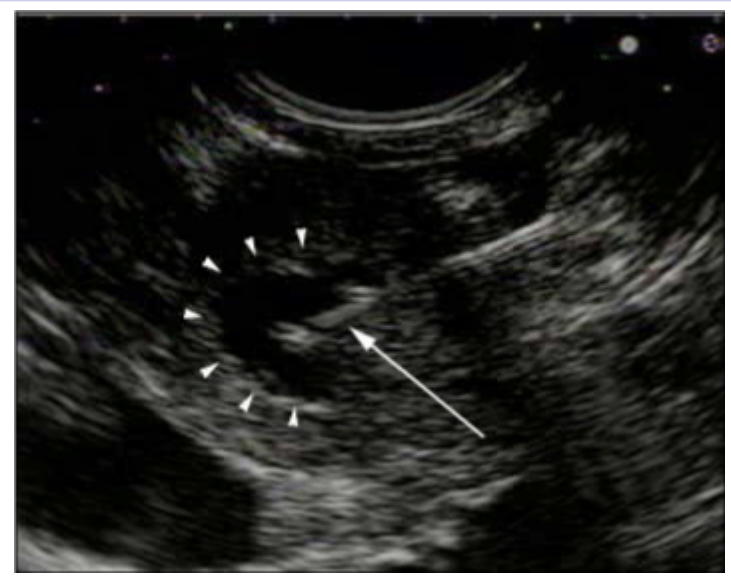

Figure 1: EUS-FNA illustrating extension of a needle (arrow) into a 2-cm pancreatic head cystic NET (arrowheads)

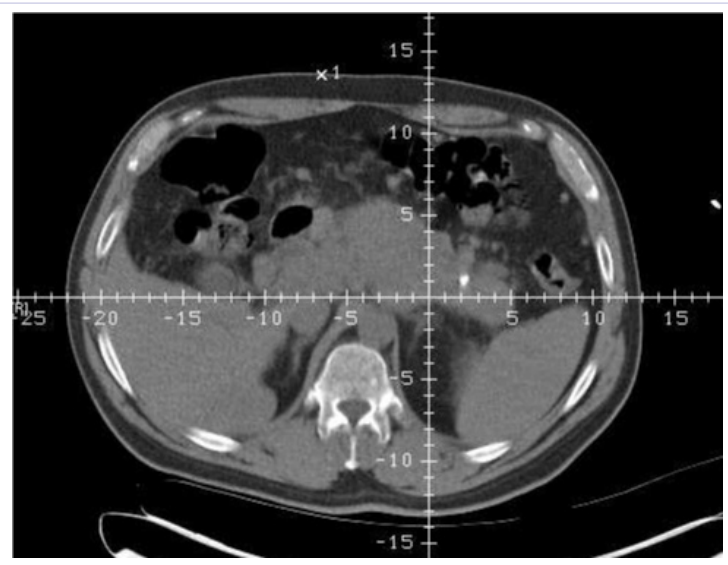

Figure 2: CT image of an 11-cm pancreatic body NET undergoing FNA. The "crosshairs" indicate the proposed location for the needle to be inserted. 


\section{Statistics}

The operating characteristics of EUS-FNA and CT-FNA including accuracy and sensitivity were calculated using SSPS 10.0 software (SSPS, Chicago, Illinois). All analyses were twotailed, and statistical significance was accepted as $p<0.05$. Because there were no true negative or false positive results by FNA, specificity could not be calculated, and positive and negative predictive values could not be compared.

\section{Results}

A total of 498 FNA procedures of pancreatic lesions were identified using the cytology database. From this database, 29 cases from 28 patients had a final diagnosis of NET by the aforementioned criteria. One patient had a repeat CTFNA procedure to evaluate residual/recurrent disease after chemotherapy; this patient was not counted twice in the data analysis. There were no FNA cases in the database in which the cytology was positive for NET but subsequent follow-up revealed a benign condition or other type of tumor. Long-term follow-up (median 18 months, range 6-132 months) was available in 25 patients.

Indications for pancreatic FNA included evaluation of a pancreatic cyst in 3 patients or a pancreatic mass in 25 patients. All 3 pancreatic cysts were evaluated by EUS-FNA whereas 16 of the pancreatic masses were evaluated by EUS-FNA, and 9 were evaluated by CT-FNA. There was no significant difference in the proportion of masses or cysts in the CT-FNA vs. EUS-FNA group $(p=0.21)$. For the 25 patients with a pancreatic mass, 5 had an underlying diagnosis of MEN-1, 5 had evidence of metastatic disease of unclear primary and 2 had symptomatic hypoglycemia of unclear etiology. There were no significant differences in associated conditions or symptoms between the two groups (Table 1).

The clinical demographics, tumor characteristics, and methods of final diagnosis for the 28 patients with pancreatic

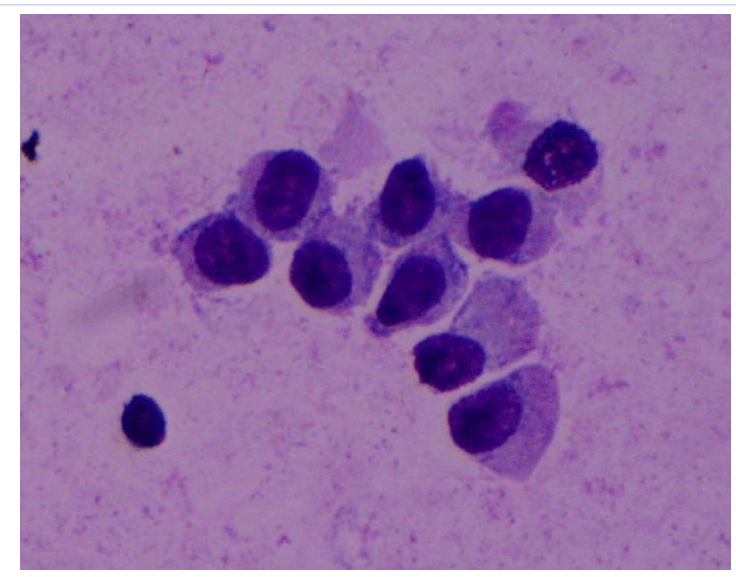

Figure 3: Typical plasmacytoid appearance of a pancreatic NET with eccentrically located nuclei and finely granular cytoplasm (Diff-Quik $600 x)$.

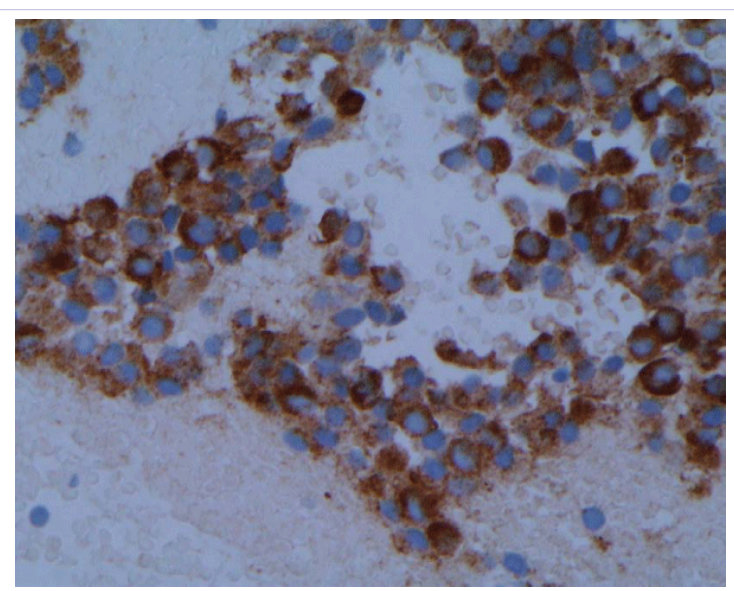

Figure 4: Immunocytochemistry of a pancreatic NET staining positive (brown) for chromogranin (400x).

Table 1: Preoperative Characteristics for Patients Undergoing FNA of a Pancreatic Mass

\begin{tabular}{|c|c|c|c|}
\hline & $\begin{array}{c}\text { CT-FNA } \\
(\mathbf{n = 9})\end{array}$ & $\begin{array}{c}\text { EUS-FNA } \\
(\mathbf{n = 1 9 )}\end{array}$ & p-value \\
\hline MEN-1 & $1(11.1 \%)$ & $4(21.1 \%)$ & 0.53 \\
\hline Metastatic disease & $3(33.3 \%)$ & $2(10.5 \%)$ & 0.15 \\
\hline Symptomatic hypoglycemia & $0(0 \%)$ & $2(10.5 \%)$ & 0.32 \\
\hline
\end{tabular}

*For the CT-FNA percentages are based on nine cases since one patient had a repeat CT-FNA.

NETs are summarized in Table 2.0f the 28 patients, 19 underwent EUS-FNA and 9 underwent CT-FNA. No patient had undergone both procedures. Among the eleven EUS-FNA cases for which the procedure report documented the presence $(n=7)$ or absence $(n=4)$ of a cytotechnologist, there was no significant difference in sensitivity for detecting pancreatic NETs with a cytotechnologist present as compared to without one $(71.4 \%$ vs. $100 \%, p=0.17$ ). In eight EUS-FNA cases, it was not specified whether a cytotechnologist was present. A cytotechnologist was present during every CT-FNA procedure. In the EUS-FNA group, the diagnosis of NET was confirmed by histopathology from the surgical and/or core biopsy specimen $(\mathrm{n}=9)$, positive FNA cytology ( $n=5)$, a history of MEN-1 $(n=3)$, and clinical course $(n=2)$. In the CT-FNA group, the final diagnosis of pancreatic NET was made by clinical course $(n=4)$, histopathology from the surgical and/or core biopsy specimen $(n=4)$, and a history of MEN-1 $(n=1)$. The patient who underwent CT-FNA twice had the final diagnosis made by the clinical course.

Performance characteristics of EUS-FNA and CT-FNA are summarized in Table 3. There was no difference in the number of passes made between the two procedural modalities. EUSFNA sampled more NETs located in the head compared to CTFNA ( $47.4 \%$ vs. $11.1 \%, p=0.017)$, and the mean size of lesions sampled by EUS-FNA was smaller $(2.7 \pm 0.9 \mathrm{~cm}$ vs. $6.5 \pm 2.1 \mathrm{~cm}$, $p=0.009$ ). There was no significant difference in sensitivity or accuracy $(73.7 \%$ vs. $88.9 \%, p=0.33)$ between EUS-FNA and CTFNA for diagnosing pancreatic NETs. 
Table 2: Clinical demographics and tumor characteristics for 28 patients with pancreatic NET

\begin{tabular}{|c|c|c|c|c|c|c|c|c|}
\hline Patient & $\begin{array}{c}\text { Age } \\
\text { (years) }\end{array}$ & Sex & $\begin{array}{c}\text { Mode } \\
\text { of FNA }\end{array}$ & Location & $\begin{array}{l}\text { Size } \\
\text { (cm) }\end{array}$ & $\begin{array}{c}\text { Cytologist } \\
\text { present? }\end{array}$ & $\begin{array}{c}\text { Cytology } \\
\text { read }\end{array}$ & $\begin{array}{c}\text { Method } \\
\text { of final } \\
\text { diagnosis }\end{array}$ \\
\hline 1 & 35 & M & CT & Head & 2.5 & Yes & ND & MEN-1 \\
\hline 2 & 75 & $\mathrm{~F}$ & СТ & Body & 2.7 & Yes & NET & $\mathrm{CC}$ \\
\hline 3 & 78 & F & CT & Body & 4 & Yes & NET & HP \\
\hline 4 & 61 & M & CT & Tail & 5 & Yes & NET & HP \\
\hline $5^{*}$ & 69 & $\mathrm{~F}$ & СT & Tail & 6 & Yes & NET & CC \\
\hline 6 & 43 & M & CT & Tail & 8 & Yes & Carcinoid & HP \\
\hline 7 & 64 & M & CT & Tail & 8.5 & Yes & NET & HP \\
\hline 8 & 42 & M & $\mathrm{CT}$ & Body & 11 & Yes & NET & $\mathrm{CC}$ \\
\hline 9 & 78 & M & CT & Tail & 11 & Yes & NET & HP \\
\hline 10 & 54 & $\mathrm{~F}$ & EUS & Body & 0.6 & NS & ND & HP \\
\hline 11 & 30 & M & EUS & Head & 0.6 & Yes & NET & MEN-1 \\
\hline 12 & 79 & $\mathrm{~F}$ & EUS & Tail & 0.9 & No & NET & Cyto \\
\hline 13 & 58 & M & EUS & Head & 1 & Yes & ND & $\mathrm{CC}$ \\
\hline 14 & 37 & $\mathrm{~F}$ & EUS & Body & 1 & Yes & NET & MEN-1 \\
\hline 15 & 42 & M & EUS & Head & 1.1 & Yes & Atypia & HP \\
\hline 16 & 60 & M & EUS & Body & 1.2 & NS & NET & Cyto \\
\hline 17 & 37 & M & EUS & Body & 1.7 & No & NET & HP \\
\hline 18 & 47 & $\mathrm{~F}$ & EUS & Tail & 1.9 & No & NET & Cyto \\
\hline 19 & 66 & F & EUS & Head & 2 & NS & Benign & HP \\
\hline 20 & 55 & M & EUS & Head & 2 & NS & NET & CC \\
\hline 21 & 21 & M & EUS & Tail & 3 & No & NET & HP \\
\hline 22 & 57 & M & EUS & Tail & 3 & NS & NET & HP \\
\hline 23 & 55 & F & EUS & Body & 4 & NS & NET & Cyto \\
\hline 24 & 56 & M & EUS & Head & 5 & NS & Benign & HP \\
\hline 25 & 37 & $\mathrm{~F}$ & EUS & Head & 5 & Yes & NET & HP \\
\hline 26 & 63 & $\mathrm{~F}$ & EUS & Head & 5 & NS & NET & Cyto \\
\hline 27 & 29 & $\mathrm{~F}$ & EUS & Tail & 6 & Yes & NET & MEN-1 \\
\hline 28 & 39 & $\mathrm{~F}$ & EUS & Head & 7 & Yes & NET & HP \\
\hline
\end{tabular}

*Patient 5 underwent CT-FNA twice. $N S$, not specified in procedure report. $N D$, non-diagnostic. $M E N-1$, mulple endocrine neoplasia, type 1. $C C$, clinical course. HP, histopathology. Cyto, cytology.

Table 3: Pancreatic NET Performance Characteristics by FNA Modality.

\begin{tabular}{|c|c|c|c|}
\hline & $\begin{array}{c}\text { EUS-FNA (n = } \\
\text { 19) }\end{array}$ & CT-FNA (n = 9)* & p-value \\
\hline Size, cm (95\% CI) & $2.7(1.8-3.6)$ & $6.5(4.4-8.6)$ & 0.009 \\
\hline Percent head lesions & $47.40 \%$ & $11.10 \%$ & 0.035 \\
\hline $\begin{array}{c}\text { Number of passes } \\
\text { (range) }\end{array}$ & $2.5(2.0-3.0)$ & $2.3(1.9-2.7)$ & 0.39 \\
\hline Sensitivity, \% (95\% CI) & $\begin{array}{c}73.7(48.6- \\
89.9)\end{array}$ & $\begin{array}{c}88.9(50.7- \\
99.4)\end{array}$ & 0.33 \\
\hline PPV, \% (95\% CI) & $100(73.2-100)$ & $100(59.8-100)$ & NA \\
\hline NPV (\%, 95\% CI) & $0(0-53.7)$ & $0(0-94.5)$ & NA \\
\hline Accuracy (\%, 95\% CI) & $\begin{array}{c}73.7(48.6- \\
89.9)\end{array}$ & $\begin{array}{c}88.9(50.7- \\
99.4)\end{array}$ & 0.33 \\
\hline
\end{tabular}

*For the CT-FNA group, size and the percent of head lesions is based on nine cases since one patient had a repeat CT-FNA. $N A$, not available.
On long-term follow-up of 25 patients, 13 had evidence of metastatic disease, including 2 patients who died from it. Twelve patients had evidence of local disease with 7 undergoing surgical resection.

\section{Discussion}

Pancreatic NETs are rare, with an incidence of 0.4 per 100,000 and comprising $1-2 \%$ of all pancreatic neoplasms [2,29]. However, despite their relative low prevalence, an increasing number of patients are being diagnosed with this condition owing to improvements in laboratory and radiological studies. A functioning NET can be diagnosed by the clinical presentation and laboratory testing. However, $15-30 \%$ of pancreatic NETs may not secrete functioning secretory products, and are discovered incidentally on imaging or as a result of mass effect producing symptoms. Because the overall prognosis is much better with pancreatic NETs as compared to adenocarcinoma, tissue confirmation of a pancreatic mass is the key to predicting the clinical course.

There are benefits of having tissue confirmation of a pancreatic NET and ruling out pancreatic adenocarcinoma in both advanced and localized disease. For advanced disease, in addition to the difference in prognosis between the two types of neoplasms, different approaches to surgery and medical management are taken. Whereas with pancreatic adenocarcinoma, in which the aims of surgical intervention are to prevent obstruction arising from the expanding lesion and to provide palliation, with locally advanced NETs a more aggressive approach, with the intent to achieve complete tumor resection, may be considered [30-33]. Furthermore, surgical resection of hepatic NET metastases may convey some survival benefit $[34,35]$. Chemotherapy for locally advanced and metastatic pancreatic adenocarcinoma includes the combination of leucovorin, 5-flurouracil, irinotecan, and oxaliplatin (FOLFIRINOX) or gemcitabine and nabpaclitaxel [36]. The choice of chemotherapeutic agent for unresectable or progressive pancreatic NETs depends on the degree of differentiation - well-differentiated NETs are treated with everolimus, sunitinib, or streptozocin- or dacarbazine-based regimens, while the combination of cisplatin and etoposide is used on poorly-differentiated disease [37].

In the case of resectable pancreatic neoplasms, a preoperative tissue diagnosis can affect the surgical approach. Adenocarcinoma localized to the head/uncinate is treated with a pancreaticoduodenectomy, and involvement of the body/ tail is treated with a distal subtotal pancreatectomy usually in combination with splenectomy [30,31]. With localized NET, the surgical approach depends on a number of tumor characteristics. Enucleation can be performed typically when there is a single, capsulated lesion, usually less than four centimeters in diameter, that does not involve the main pancreatic duct [38]. In all other situations of localized NETs, the standard procedures are pancreaticoduodenectomy for head/uncinate lesions and distal pancreatectomy without splenectomy for body/tail lesions [39-41]. In patients with MEN-1, pancreatic NETs are usually multifocal, and intraoperative ultrasound is necessary $[32,42]$. 
Localized disease in this case is treated with a distal subtotal pancreatectomy and enucleation of any tumors found in the head $[33,35]$.

To our knowledge, only one prior study has looked at EUSFNA and CT-FNA in the evaluation of endocrine tumors of the pancreas [27]. However, this smaller study's objective was to compare the cytologic features of pancreatic islet cell tumors and specimen adequacy, and it did not make any direct statistical comparison between the two FNA modalities. This current study is the first to compare the operating characteristics of EUSFNA and CT-FNA, the two most commonly used non-surgical modalities used to obtain tissue from pancreatic lesions, in the diagnosis of pancreatic NETs. We demonstrate comparable sensitivity and accuracy between the two FNA methods, similar to studies evaluating pancreatic lesions as a whole [5,6]. A notable finding in our study was that the mean size of pancreatic NETs sampled by EUS-FNA was significantly smaller than that of CT-FNA. Furthermore, almost half of NETs evaluated by EUSFNA were located in the head region, whereas CT-FNA evaluated head NETs in just over ten percent of the cases. These findings suggest that EUS-FNA may be advantageous over CT-FNA in that it can be used to accurately diagnose smaller NETs that tend to be located in the pancreatic head. This inference is supported by the findings of Mallery et al. when they compared tissue sampling of all types of pancreatic mass lesions by EUS, CT/ultrasound, and surgery, and found that the mean size of the sampled masses were significantly smaller in the EUS-FNA group [5]. Although not statistically significant, they also noted that the EUS group had a higher proportion of head lesions compared to the CT/ ultrasound and surgery groups.

This finding of EUS-FNA being potentially advantageous over CT-FNA in the diagnosis of smaller NETs in the pancreatic head has particular implications in the diagnosis and treatment of MEN1. MEN-1 patients with pancreatic NETs may have multiple small tumors existing throughout the pancreas rather than a single lesion [32]. Precise determination of disease extent and accurate cytologic diagnosis is essential in these scenarios as it may significantly affect therapeutic approach. These smaller lesions may be completely missed on conventional imaging, inaccessible on CT-FNA, or inaccurately diagnosed via the aforementioned approach, leading to suboptimal treatment. As mentioned earlier, EUS is the preferred modality in the pre-operative localization of these smaller multifocal tumors and has the added advantage of being able to use EUS-FNA to obtain a cytologic diagnosis [4]. Our results suggest that while not $100 \%$ sensitive, EUS-FNA can accurately diagnose these smaller lesions, especially if they are located in the pancreatic head. Thus we recommend that EUSFNA be the preferred approach in the workup of pancreatic NETs in MEN-1 given the risk of multifocal disease.

There are a few limitations in our study design that warrant mention. First, this is a retrospective study of an uncommon disease; therefore, we could not control for a number of variables when comparing the two FNA methods. From the medical records, it is not clear why one FNA method was chosen over the other for any particular patient. There were also no significant differences in the preoperative characteristics of each group. However, more CT-FNAs occurred earlier in the study, and more EUS-FNAs occurred later as this technology became more easily accessible. It is also possible that pre-procedural localization of the mass in the pancreatic head instead of the tail prompted preferential selection of EUS-FNA over CT-FNA as the closer proximity of the pancreatic head to the enteric lumen relative to the tail could theoretically allow easier access via EUS-FNA over CT-FNA. The second limitation is the varied skill and experience of the endosonographers and radiologists performing the study. EUS and EUS-FNA results are highly operator dependent, and performance by an inexperienced endosonographer can result in less reliable results. In our study, all procedures were performed by experienced endosonographers, so our results may only be applicable to other institutions possessing EUS expertise. Third, since many patients were referred from outside our institution, obtaining complete follow-up in our patients was limited. Eight of the 19 patients who underwent EUS-FNA had their final diagnosis of pancreatic NET based on the FNA itself. At our institution, false-positive specimens on cytology are rare. Based on this fact and a prior study by Fritscher-Ravens et al, we assumed 100\% specificity for the FNA procedure, and we used this criterion for a final diagnosis when no other means of confirming the cytologic diagnosis was available [28]. This is not the ideal method for confirming a neoplastic process, as histopathology and the patient's clinical course generally are more accepted means of diagnosis. Finally, documentation of whether a cytotechnologist was present during EUS-FNA occurred in only 11 of the 19 cases. This small number makes it difficult to interpret the effect that a cytotechnologist may have had on the accuracy of the procedure. Our limited data would suggest, counterintuitively, that the accuracy is higher when one is not present.

In our study, FNA results including suspicious, atypia, and non-diagnostic were grouped together as negative for NET. Given that there was a relatively high false-negative rate for each FNA method, the results of the FNA should only be used clinically if there is positive diagnosis of NET. A non-NET finding on FNA, when there is a strong clinical suspicion of NET, should prompt further workup, including repeat biopsy and, in cases of localized disease seen on imaging, exploratory surgery. Furthermore, in addition to specific treatment of the NET, patients not previously known to have MEN-1 who are diagnosed with a pancreatic NET should undergo a work-up to evaluate for this syndrome due to the high frequency of pancreatic NETs in patients with MEN-1 [2]. This study suggests that the smaller tumor sizes and multifocal lesions observed in MEN-1patients are more amenable to visualization and confirmation of histological grade via EUS-FNA.

In summary, our study shows that EUS-FNA and CT-FNA have comparable sensitivity for diagnosing pancreatic NETs. The main advantage of EUS-FNA may be its ability to accurately diagnose smaller NETs that tend to be located in the head of the pancreas. The results of FNA should only guide further clinical steps if there is a positive diagnosis of NET and patients diagnosed with 
pancreatic NETs should undergo workup for possible MEN-1. Future studies in a larger group of patients are warranted to confirm our initial findings and to determine if the presence of a cytotechnologist during the EUS-FNA procedure can improve the diagnostic sensitivity of EUS-FNA for pancreatic NETs.

\section{Acknowledgement}

This work received grant support from: Department of Veterans Affairs RR\&D Merit Review (JRP) I01 RX000194; Human Studies CORE through CURE: Digestive Diseases Research Center supported by NIH grant P30DK41301; NIH Training Grant NIDDK T32 (JNB).

\section{References}

1. Modlin IM, Tang LH. Approaches to the diagnosis of gut neuroendocrine tumors: the last word (today). Gastroenterology. 1997;112(2):583590.

2. Oberg K, Eriksson B. Endocrine tumours of the pancreas. Clinical gastroenterology 2005;19(5):753-781.

3. Jemal A, Murray T, Ward E, Samuels A, Tiwari RC, Ghafoor A, et al Cancer statistics, 2005. CA Cancer J Clin. 2005;55(1):10-30.

4. Gines A, Vazquez-Sequeiros E, Soria MT, Clain JE, Wiersema MJ. Usefulness of EUS-guided fine needle aspiration (EUS-FNA) in the diagnosis of functioning neuroendocrine tumors. Gastrointestinal endoscopy. 2002;56(2):291-296.

5. Mallery JS, Centeno BA, Hahn PF, Chang Y, Warshaw AL, Brugge WR Pancreatic tissue sampling guided by EUS, CT/US, and surgery: a comparison of sensitivity and specificity. Gastrointestinal endoscopy. 2002;56(2):218-224

6. Volmar KE, Vollmer RT, Jowell PS, Nelson RC, Xie HB. Pancreatic FNA in 1000 cases: a comparison of imaging modalities. Gastrointestinal endoscopy. 2005;61(7):854-861.

7. Antillon MR, Chang KJ. Endoscopic and endosonography guided fineneedle aspiration. Gastrointest Endosc Clin N Am. 2000;10(4):619636.

8. Baron PL, Aabakken LE, Cole DJ, LeVeen MB, Baron LF, Daniel DM, et al. Differentiation of benign from malignant pancreatic masses by endoscopic ultrasound. Annals of surgical oncology. 1997;4(8):639643.

9. Bentz JS, Kochman ML, Faigel DO, Ginsberg GG, Smith DB, Gupta PK Endoscopic ultrasound-guided real-time fine-needle aspiration: clinicopathologic features of 60 patients. Diagnostic cytopathology. 1998;18(2):98-109.

10. Bhutani MS, Hawes RH, Baron PL, Sanders-Cliette A, van Velse A, Osborne JF, et al. Endoscopic ultrasound guided fine needle aspiration of malignant pancreatic lesions. Endoscopy. 1997;29(9):854-858.

11. Chang KJ, Albers CG, Erickson RA, Butler JA, Wuerker RB, Lin F. Endoscopic ultrasound-guided fine needle aspiration of pancreatic carcinoma. The American journal of gastroenterology. 1994;89(2):263-266

12. Chang KJ, Nguyen P, Erickson RA, Durbin TE, Katz KD. The clinical utility of endoscopic ultrasound-guided fine-needle aspiration in the diagnosis and staging of pancreatic carcinoma. Gastrointestinal endoscopy. 1997;45(5):387-393.
13. Chhieng DC, Jhala D, Jhala N, Isam Eltoumet, Victor K. Chen, Selwyn Vickers, et al. Endoscopic ultrasound-guided fine-needle aspiration biopsy: a study of 103 cases. Cancer. 2002;96(4):232-239.

14. Di Stasi M, Lencioni R, Solmi L, Magnolfi F, Caturelli E, De Sio I, et al. Ultrasound-guided fine needle biopsy of pancreatic masses: results of a multicenter study. Am J Gastroenterol. 1998;93(8):1329-1333.

15. Eloubeidi MA, Jhala D, Chhieng DC, et al. Yield of endoscopic ultrasound-guided fine-needle aspiration biopsy in patients with suspected pancreatic carcinoma. Cancer. 2003;99(5):285-292.

16. Giovannini M, Seitz JF, Monges G, Perrier H, Rabbia I. Fine-needle aspiration cytology guided by endoscopic ultrasonography: results in 141 patients. Endoscopy. 1995;27(2):171-177.

17. Palazzo L, Roseau G, Gayet B, Vilgrain V, Belghiti J, Fékéte F, et al. Endoscopic ultrasonography in the diagnosis and staging of pancreatic adenocarcinoma. Results of a prospective study with comparison to ultrasonography and CT scan. Endoscopy. 1993;25(2):143-150.

18. Raut CP, Grau AM, Staerkel GA, Kaw M, Tamm EP, Wolff RA, et al. Diagnostic accuracy of endoscopic ultrasound-guided fine-needle aspiration in patients with presumed pancreatic cancer. J Gastrointest Surg. 2003;7(1):118-126.

19. Rosch T, Lorenz R, Braig C, Feuerbach S, Siewert JR, Schusdziarra V, et al. Endoscopic ultrasound in pancreatic tumor diagnosis. Gastrointest Endosc. 1991;37(3):347-352.

20. Shin HJ, Lahoti S, Sneige N. Endoscopic ultrasound-guided fine-needle aspiration in 179 cases. Cancer. 2002;96(3):174-180.

21. Suits J, Frazee R, Erickson RA. Endoscopic ultrasound and fine needle aspiration for the evaluation of pancreatic masses. Arch Surg. 1999;134(6):639-642.

22.Voss M, Hammel P, Molas G, Palazzo L, Dancour A, O’Toole D, et al. Value of endoscopic ultrasound guided fine needle aspiration biopsy in the diagnosis of solid pancreatic masses. Gut. 2000;46(2):244-249.

23. Wiersema MJ, Vilmann P, Giovannini M, Chang KJ, Wiersema LM. Endosonography-guided fine-needle aspiration biopsy: diagnostic accuracy and complication assessment. Gastroenterology. 1997;112(4):1087-1095.

24. Williams DB, Sahai AV, Aabakken L, I Penman, A van Velse, J Webb, et al. Endoscopic ultrasound guided fine needle aspiration biopsy: a large single centre experience. Gut. 1999;44(5):720-726.

25. Ardengh JC, de Paulo GA, Ferrari AP. EUS-guided FNA in the diagnosis of pancreatic neuroendocrine tumors before surgery. Gastrointestinal endoscopy. 2004;60(3):378-384.

26. Chatzipantelis P, Salla C, Konstantinou P, Karoumpalis I, Sakellariou S, Doumani I. Endoscopic ultrasound-guided fine-needle aspiration cytology of pancreatic neuroendocrine tumors: a study of 48 cases. Cancer. 2008;114(4):255-262. doi: 10.1002/cncr.23637

27. Jhala D, Eloubeidi M, Chhieng DC, Frost A, Eltoum IA, Roberson J, et al. Fine needle aspiration biopsy of the islet cell tumor of pancreas: a comparison between computerized axial tomography and endoscopic ultrasound-guided fine needle aspiration biopsy. Ann Diagn Pathol. 2002;6(2):106-112.

28. Fritscher-Ravens A, Izbicki JR, Sriram PV, Christina Krause, W Trudo Knoefel, Theodoros Topalidis, et al. Endosonographyguided, fine-needle aspiration cytology extending the indication for organ-preserving pancreatic surgery. The American journal of gastroenterology. 2000;95:2255-2260. doi:10.1111/j.15720241.2000.02311.x 
29. Eriksson B, Oberg K. Neuroendocrine tumours of the pancreas. The British journal of surgery. 2000;87(2):129-131. DOI: 10.1046/j.13652168.2000.01277.x

30. Goldstein D, Carroll S, Apte M, Keogh G. Modern management of pancreatic carcinoma. Intern Med J. 2004;34(8):475-481.

31. Wray CJ, Ahmad SA, Matthews JB, Lowy AM. Surgery for pancreatic cancer: recent controversies and current practice. Gastroenterology. 2005;128(6):1626-1641.

32. Kouvaraki MA, Solorzano CC, Shapiro SE, Yao JC, Perrier ND, Lee JE, et al. Surgical treatment of non-functioning pancreatic islet cell tumors. J Surg Oncol. 2005;89(3):170-185.

33. Akerstrom G, Hessman O, Skogseid B. Timing and extent of surgery in symptomatic and asymptomatic neuroendocrine tumors of the pancreas in MEN 1. Langenbecks Arch Surg. 2002;386(8):558-569.

34. Norton JA. Endocrine tumours of the gastrointestinal tract. Surgical treatment of neuroendocrine metastases. Best Pract Res Clin Gastroenterol. 2005;19(4):577-583.

35. Falconi M, Bettini R, Boninsegna L, Crippa S, Butturini G, Pederzoli P. Surgical strategy in the treatment of pancreatic neuroendocrine tumors. JOP. 2006;7(1):150-156.

36. Ryan DP, Hong TS, Bardeesy N. Pancreatic adenocarcinoma. The New
England journal of medicine. 2014;371:1039-1049. DOI: 10.1056/ NEJMra1404198

37. Kunz PL, Reidy-Lagunes D, Anthony LB, Bertino EM, Brendtro K, Chan $\mathrm{JA}$, et al. Consensus guidelines for the management and treatment of neuroendocrine tumors. Pancreas. 2013;42(4):557-77. doi: 10.1097/ MPA.0b013e31828e34a4

38. Park BJ, Alexander HR, Libutti SK, Huang J, Royalty D, Skarulis MC, et al. Operative management of islet-cell tumors arising in the head of the pancreas. Surgery. 1998;124(6):1056-1061.

39. Delcore R, Friesen SR. Role of pancreatoduodenectomy in the management of primary duodenal wall gastrinomas in patients with Zollinger-Ellison syndrome. Surgery. 1993;112(6):1016-1022.

40. Phan GQ, Yeo CJ, Hruban RH, Littemoe KD, Pitt HA, Cameron JL. Surgical experience with pancreatic and peripancreatic neuroendocrine tumors: Review of 125 patients. J Gastrointest Surg. 1998;2(5):473482. doi: 10.1016/S1091-255X(98)80039-5

41. Jordan PH Jr. A personal experience with pancreatic and duodenal neuroendocrine tumors. J Am Coll Surg. 1999;189(5):470-482.

42. Thompson NW. Current concepts in the surgical management of multiple endocrine neoplasia type 1 pancreatic-duodenal disease. Results in the treatment of 40 patients with Zollinger-Ellison syndrome, hypoglycaemia or both. J Intern Med. 1998;243(6):495500 . 